\title{
Additional value of lateral tissue Doppler imaging in the assessment of diastolic dysfunction among subjects with pseudonormal pattern of mitral inflow
}

\author{
Hack-Lyoung Kim', Joo-Hee Zo ${ }^{1,3^{*}}$, Jae-Bin Seo ${ }^{1}$, Woo-Young Chung ${ }^{1}$, Yong-Jin Kim², Sang-Hyun Kim',
} Myung-A Kim ${ }^{1}$ and Dae-Won Sohn ${ }^{2}$

\begin{abstract}
Background: There has been a lack of research on further stratification of subjects who have pseudonormal pattern of mitral inflow. The study aim was to clarify 2 different groups with different diastolic function grades among these subjects using lateral tissue Doppler imaging (TDI).
\end{abstract}

Methods: A total of 122 consecutive subjects showing pseudonormal pattern of mitral inflow (E/A $\geq 1$ and septal $\left.\mathrm{e}^{\prime} / \mathrm{a}^{\prime}<1\right)$ without structural abnormality were prospectively recruited. TDI measurements were performed from both septal and lateral mitral annuli.

Results: Study subjects were stratified according to lateral TDI pattern ( $\left(e^{\prime} / a^{\prime}<1[n=50]\right.$ versus $e^{\prime} / a^{\prime} \geq 1[n=72]$ ). Subjects with lateral $\mathrm{e}^{\prime} / \mathrm{a}^{\prime}<1$ had higher values of left atrial volume index (LAVI) and E/e' compared to those for lateral $e^{\prime} / a^{\prime} \geq 1$ ( $p<0.001$ for each). Among subjects with lateral $e^{\prime} / a^{\prime} \geq 1$, only $9.3 \%$ of subjects had grade II diastolic dysfunction, whereas among subjects with lateral $e^{\prime} / a^{\prime}<1$, majority of subjects (64.1\%) had grade II diastolic dysfunction $(p<0.001)$. Multiple linear regression analysis showed that lateral $e^{\prime} / a^{\prime}$ was independently associated with LAVI $(\beta=-0.484, p<0.001)$, even after adjusting for potential confounders including age, sex, body mass index, hypertension and diabetes.

Conclusions: In subjects without structural abnormality showing $E / A \geq 1$ and septal $e^{\prime} / a^{\prime}<1$, lateral TDI measurement is useful in the assessment of diastolic dysfunction. Lateral $e^{\prime} / a^{\prime} \geq 1$ is a valuable indicator of early diastolic dysfunction but not of advanced diastolic dysfunction in this population.

Keywords: Diastolic dysfunction, Mitral annulus, Pseudonormal pattern, Tissue Doppler imaging, Transthoracic echocardiography

\section{Background}

Evaluation of left ventricular (LV) diastolic function is an essential component of routine echocardiographic examination. As the clinical significance of heart failure with preserved ejection fraction (HFpEF) has increased [1,2], information on LV diastolic function and filling pressure

\footnotetext{
* Correspondence: jooheezo@hanmail.net

${ }^{1}$ Cardiovascular Center, Seoul National University Boramae Medical Center, Seoul, Korea

${ }^{3}$ Department of Internal Medicine, Seoul National University Boramae Medical Center, Seoul National University College of Medicine, 39 Boramae-gil, Dongjak-gu, Seoul 156-707, Korea

Full list of author information is available at the end of the article
}

is of great importance to clinicians [3]. Doppler analysis of mitral inflow and pulmonary vein flow has been the main clinical tool used to evaluate LV diastolic function. However, this analysis has several shortcomings. It is dependent on loading conditions such as volume status and left atrial (LA) pressure, and is difficult to obtain in patients for whom the image qualities are poor. More recently, tissue Doppler imaging (TDI), which is relatively independent of loading conditions and image quality, has been found to be useful in the assessment of LV diastolic function $[4,5]$. In addition, the ratio between early diastolic mitral inflow (E) and early diastolic mitral

\section{Biomed Central}

(c) 2013 Kim et al.; licensee BioMed Central Ltd. This is an Open Access article distributed under the terms of the Creative Commons Attribution License (http://creativecommons.org/licenses/by/2.0), which permits unrestricted use, distribution, and reproduction in any medium, provided the original work is properly cited. 
annular tissue velocity (e') was found to correlate well with LV filling pressure, and are now widely used to diagnose diastolic dysfunction [5-7]. Moreover, combined information of mitral inflow and TDI is able to differentiate between normal and pseudonormal diastolic dysfunction [5].

Previously, we have shown that some people have discrepancies in mitral annular velocity patterns between the septal and lateral annulus because the septal annular velocity more rapidly decreases with age, when compared with that of lateral annulus [8]. However, the clinical implications of these findings have not been further studied. The present study focused on subjects with ratios of peak early to late mitral inflow wave velocity $[\mathrm{E} / \mathrm{A}] \geq 1$ and ratios of peak septal early to late diastolic mitral annular tissue velocity $\left[\mathrm{e}^{\prime} / \mathrm{a}^{\prime}\right]<1$, showing pseudonormal pattern of mitral inflow $[5,6]$. These subjects are frequently considered to have an advanced stage of diastolic dysfunction. Among these subjects, we have noticed 2 different groups with different severities of diastolic dysfunction according to the lateral TDI patterns: one group with lateral e' $/ \mathrm{a}^{\prime}<1$ (concordant to septal TDI) and the other group with lateral e'/a' $\geq 1$ (discordant to septal TDI) (Figure 1). This study describes different characteristics between these 2 groups and suggests additional benefit of measuring lateral TDI in the evaluation of diastolic function in this population.

\section{Methods}

Study subjects

This is a single center study performed at Seoul National University Boramae Medical Center (Seoul, Korea). Between March and October 2012, a total of 661 consecutive subjects in sinus rhythm without significant structural abnormalities in transthoracic echocardiographic (TTE) examination were prospectively recruited. Subjects with LV ejection fraction $<50 \%$, valvular stenosis or regurgitation of more than mild degree, regional wall motion abnormalities, LV hypertrophy, mitral annular calcification, prosthetic heart valve, and intra-cardiac shunt or pericardial effusion were excluded from the study. Subjects with poor sonic window or poor cooperation during the exam were also excluded. These 661 subjects were grouped according to mitral inflow patterns: a group with $\mathrm{E} / \mathrm{A} \geq 1(\mathrm{n}=385)$ and the other group with $\mathrm{E} / \mathrm{A}<1(\mathrm{n}=276)$. Among 385 subjects with $\mathrm{E} / \mathrm{A} \geq 1$, the present study focused on 122 subjects with septal $\mathrm{e}^{\prime} / \mathrm{a}$ ' $<1$, who were frequently considered to have a pseudonormalization suggesting advanced stage of diastolic dysfunction [5,6]. From clinical experience, we have noticed that these subjects can be stratified into 2 groups with different diastolic dysfunction severities by the lateral TDI pattern. In order to investigate the usefulness of lateral TDI in the assessment of diastolic function, these 122 subjects were then classified according to
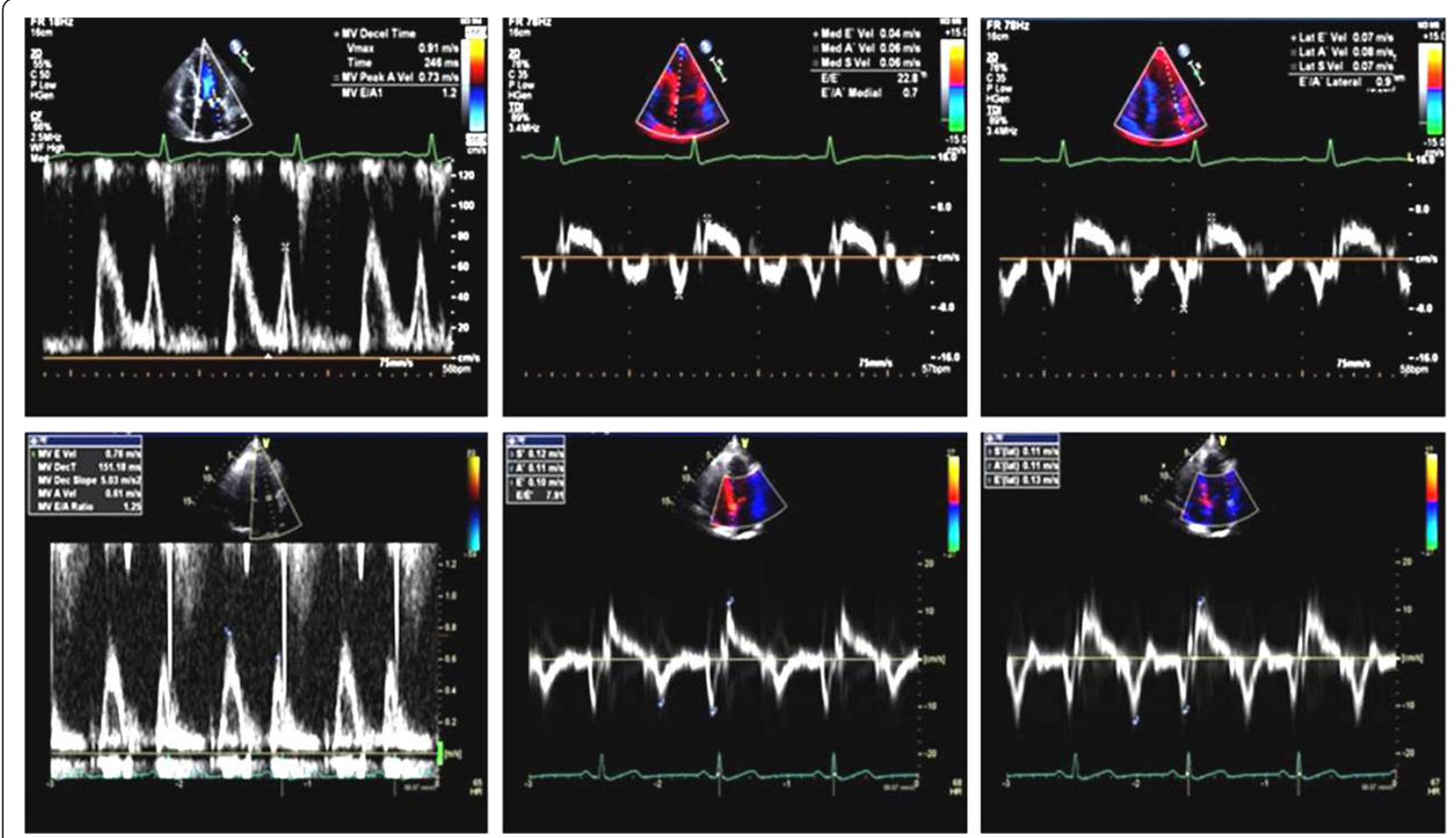

Figure 1 Representative figures of a subject with concordance (upper 3 figures) and discordance pattern (lower 3 figures) of tissue Doppler imaging. 
the pattern of lateral TDI. One group of subjects had lateral $\mathrm{e}^{\prime} / \mathrm{a}$ ' $<1$, and designated as "concordance (to medial TDI pattern) group" ( $\mathrm{n}=50)$. The other group had lateral $\mathrm{e}^{\prime} / \mathrm{a}$ ' $\geq 1$, and this group was designated as "discordance (to medial TDI pattern) group" ( $n=72)$. Study flow for enrollment is shown in Figure 2. Approval of the study protocol was obtained from the Institutional Review Board of Seoul National University Boramae Medical Center (Seoul, Korea). Informed consent was waived due to the routine nature of the information collected.

\section{Echocardiography}

TTE was performed according to the recommendations of current guidelines [9-11] using commercially available equipments (Sequoia, Siemens Medical Solutions and Vivid 7, GE Medical Systems) in the left lateral decubitus position. M-mode echocardiographic features were used to measure LV and LA dimensions, with LV ejection fraction from the parasternal short axis view at the mitral chordae level. LA volume was calculated using the prolate ellipsoid method [9]. Peak early transmitral filling velocities during early diastole (E), late diastole (A) and deceleration time (DT) were imaged in the apical four chamber view at the tip of mitral leaflets. Color-coded TDI was applied to the apical four chamber view to determine the mean early (e') and late (a') velocities at both septal and lateral mitral annuli. Two experienced cardiosonographers performed the echocardiography. Interobserver agreement for lateral E/e' was evaluated by Spearman's correlation among 50 subjects. The correlation coefficient was 0.905 in our laboratory.

\section{LV diastolic function grade}

LV diastolic function was graded according to the current guidelines [10]. Normal LV diastolic function was defined as septal $\mathrm{e}^{\prime} \geq 8 \mathrm{~cm} / \mathrm{s}$ and LA volume index

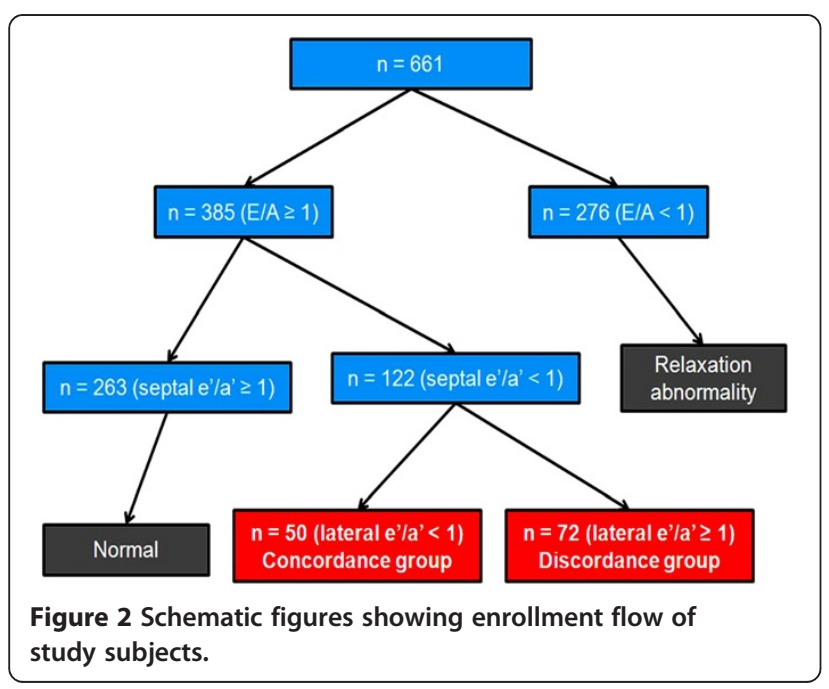

$(\mathrm{LAVI})<34 \mathrm{ml} / \mathrm{m}^{2}$. Grade II diastolic dysfunction was defined as septal $\mathrm{e}^{\prime}<8 \mathrm{~cm} / \mathrm{s}, \mathrm{LAVI} \geq 34 \mathrm{ml} / \mathrm{m}^{2}$, DT $160-200$ $\mathrm{ms}$ and septal $\mathrm{E} / \mathrm{e}^{\prime} \geq 9$. Because we had only enrolled subjects with $\mathrm{E} / \mathrm{A} \geq 1$, there were no subjects with grade I diastolic dysfunction, which is defined as $\mathrm{E} / \mathrm{A}<0.8$. Grade III diastolic dysfunction was defined as septal e' $<8 \mathrm{~cm} / \mathrm{s}$, LAVI $\geq 34 \mathrm{ml} / \mathrm{m}^{2}, \mathrm{E} / \mathrm{A} \geq 2, \mathrm{DT}<160 \mathrm{~ms}$ and septal E/e' $\geq$ 13. There were no subjects with grade III diastolic dysfunction in the study.

\section{Statistical analysis}

Continuous variables were presented as mean \pm standard deviation (SD), and categorical variables were expressed as percentages. Continuous variables were compared using the Student $t$ test, and categorical variables were compared using the Chi-square test. Pearson's correlation method was used to assess the relationship between two continuous variables. Multiple linear regression analysis was performed to assess independent association between LAVI and lateral e'/a' by adjusting for age, sex, body mass index and history of hypertension and diabetes. To identify the best cut-off value of lateral e'/a' as a predictor of LAVI $\geq 34 \mathrm{ml} / \mathrm{m}^{2}$ [10], receiver operating characteristic (ROC) curve analysis was used. A $p$-value $<0.05$ was considered statistically significant. All statistical analyses were conducted using SPSS 18.0 (Chicago, IL, USA).

\section{Results}

The baseline characteristics of the study subjects are summarized in Table 1. The mean age was $56.0 \pm 13.2$ years, and 59 (48.4\%) subjects were men. The prevalence of hypertension, diabetes and dyslipidemia were 41.8, 13.9 and $15.6 \%$, respectively.

\section{Comparisons between concordance and discordance groups}

The results of comparative analyses between the concordance and discordance groups are also shown in Table 1. Subjects in the concordance group were older $(59.6 \pm 14.1$ versus $53.5 \pm 12.0$ years, $p=0.012)$. Otherwise, there were no significance differences in terms of cardiovascular risk factors including sex, body mass index, hypertension, diabetes and dyslipidemia between the 2 groups ( $p>0.05$ for each). LV end-diastolic (LVEDD) and end-systolic dimensions (LVESD) were greater for the concordance group than those of discordance group ( $p<0.05$ for each). LAVI was significantly higher for the concordance group than that for the discordance group $\left(26.4 \pm 8.8\right.$ versus $17.9 \pm 6.6 \mathrm{ml} / \mathrm{m}^{2}$, $p<0.001)$. Septal e' wave velocity was significantly lower $(6.4 \pm 1.7$ versus $7.5 \pm 1.6 \mathrm{~cm} / \mathrm{s}, p<0.001)$ and $\mathrm{E} / \mathrm{e}$ ' ratios were significantly higher $(14.5 \pm 6.4$ versus $10.7 \pm 3.5, p<0.001)$ in the concordance groups than those in the discordance group. 
Table 1 Comparisons of characteristics among study subjects

\begin{tabular}{|c|c|c|c|c|}
\hline Characteristic & All $(n=122)$ & Concordance group $(n=50)$ & Discordance group $(n=72)$ & $p^{*}$ \\
\hline Age, years & $56.0 \pm 13.2$ & $59.6 \pm 14.1$ & $53.5 \pm 12.0$ & 0.012 \\
\hline Male sex, n (\%) & $59(48.4)$ & $23(46.0)$ & $36(50.0)$ & 0.664 \\
\hline $\mathrm{BMl}, \mathrm{kg} / \mathrm{m}^{2}$ & $25.2 \pm 4.1$ & $25.7 \pm 4.1$ & $24.6 \pm 4.2$ & 0.336 \\
\hline Hypertension, n (\%) & $51(41.8)$ & $24(48.0)$ & $27(37.5)$ & 0.248 \\
\hline Diabetes, n (\%) & $17(13.9)$ & $10(20.0)$ & $7(9.7)$ & 0.107 \\
\hline Dyslipidemia, n (\%) & 19 (15.6) & $12(16.7)$ & $7(14.0)$ & 0.690 \\
\hline \multicolumn{5}{|l|}{ Echocardiographic parameters } \\
\hline LVESD, mm & $29.6 \pm 3.6$ & $30.7 \pm 3.4$ & $28.8 \pm 3.5$ & 0.004 \\
\hline LVEDD, mm & $48.0 \pm 4.3$ & $49.5 \pm 3.0$ & $47.4 \pm 3.0$ & $<0.001$ \\
\hline LVEF, \% & $68.1 \pm 5.0$ & $67.4 \pm 5.8$ & $68.6 \pm 4.2$ & 0.213 \\
\hline LVSWT, mm & $9.3 \pm 1.4$ & $9.5 \pm 1.7$ & $9.2 \pm 1.3$ & 0.234 \\
\hline LVPWT, mm & $9.2 \pm 1.3$ & $9.2 \pm 1.6$ & $9.1 \pm 1.0$ & 0.563 \\
\hline LA dimension, mm & $36.7 \pm 5.2$ & $39.0 \pm 5.3$ & $35.2 \pm 4.6$ & $<0.001$ \\
\hline $\mathrm{LA}$ volume index, $\mathrm{ml} / \mathrm{m}^{2}$ & $21.4 \pm 8.6$ & $26.4 \pm 8.8$ & $17.9 \pm 6.6$ & $<0.001$ \\
\hline E wave velocity, $\mathrm{cm} / \mathrm{s}$ & $80 \pm 16$ & $84 \pm 19$ & $77 \pm 13$ & 0.027 \\
\hline A wave velocity, $\mathrm{cm} / \mathrm{s}$ & $64 \pm 14$ & $68 \pm 16$ & $61 \pm 12$ & 0.009 \\
\hline Deceleration time, ms & $205 \pm 36$ & $208 \pm 41$ & $202 \pm 32$ & 0.384 \\
\hline Septal e' velocity, $\mathrm{cm} / \mathrm{s}$ & $7.1 \pm 1.7$ & $6.4 \pm 1.7$ & $7.5 \pm 1.6$ & $<0.001$ \\
\hline Septal a' velocity, $\mathrm{cm} / \mathrm{s}$ & $9.5 \pm 2.1$ & $9.3 \pm 2.1$ & $9.7 \pm 2.0$ & $<0.001$ \\
\hline Lateral $\mathrm{e}^{\prime}$ velocity, $\mathrm{cm} / \mathrm{s}$ & $10.0 \pm 2.6$ & $8.2 \pm 2.0$ & $11.1 \pm 2.1$ & 0.027 \\
\hline Lateral a' velocity, $\mathrm{cm} / \mathrm{s}$ & $9.6 \pm 2.2$ & $10.6 \pm 2.5$ & $8.9 \pm 1.8$ & 0.009 \\
\hline E/e' (septal) & $12.2 \pm 5.2$ & $14.5 \pm 6.4$ & $10.7 \pm 3.5$ & $<0.001$ \\
\hline
\end{tabular}

$B M I$ body mass index, LVESD left ventricular end-systolic dimension, LVEDD left ventricular end-diastolic dimension, $L V E F$ left ventricular ejection fraction, LVSWT left ventricular septal wall thickness, LVPWT left ventricular posterior wall thickness, LA left atrium.

"significance from the result of comparison between concordance and discordance groups.

The value of lateral e'/a' for discrimination of normal from grade II diastolic dysfunction

According to current guidelines [10], study subjects were grouped into normal and grade II diastolic dysfunction. If big discrepancies existed between parameters for diastolic dysfunction grading, this group of subjects was regarded as undetermined. The number of subjects in the normal, grade II and undetermined groups was 53 (43\%), 29 (24\%) and 40 (33\%), respectively. Among subjects with lateral $e^{\prime} / a^{\prime} \geq 1$, only 4 subjects (9.3\%) had grade II diastolic dysfunction, whereas among subjects with lateral $\mathrm{e}^{\prime} / \mathrm{a}$ ' $<1$, the majority of subjects $(\mathrm{n}=25$, $64.1 \%)$ had grade II diastolic dysfunction $(p<0.001)$ (Figure 3).

Independent association between LAVI and lateral e'/a' LAVI is a useful indicator of LV diastolic function [12]. We tested whether lateral e'/a' had an association with LAVI. In a simple correlation analysis, lateral e'/a' was significantly associated with LAVI: increases in lateral e'/a' was associated with decreases in LAVI $(r=-0.469$, $p<0.001$ ) (Figure 4A). It is well known that Doppler tissue e' velocity decreases with age [13]. Therefore, age was a powerful confounder, and this was addressed in this study. As expected, lateral e'/a' had a significant correlation with age, and decreased with advancing age $(r=-0.429, p<0.001)$ (Figure 4B).

Subsequently, potential confounders, including age, were addressed using a multivariable model. A multiple

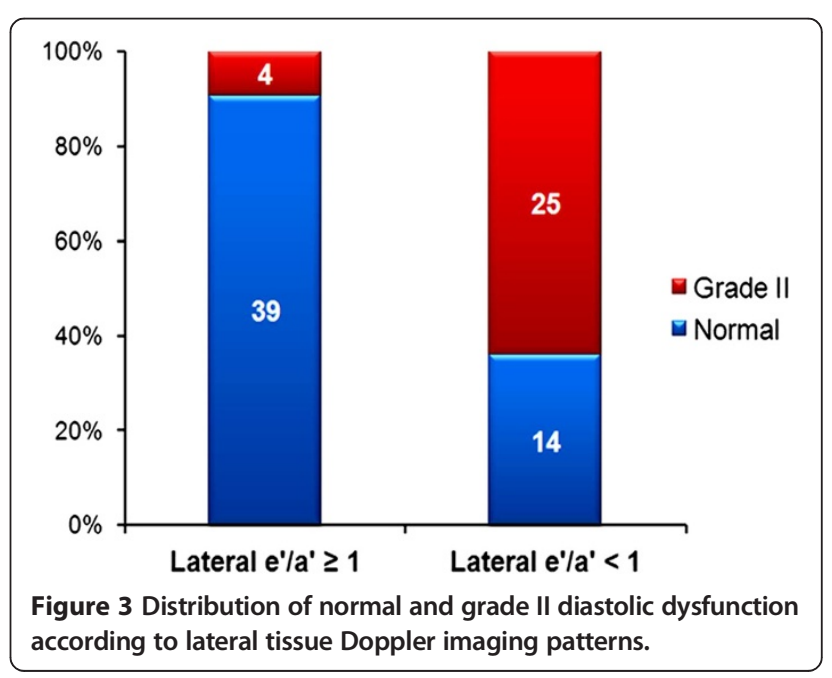



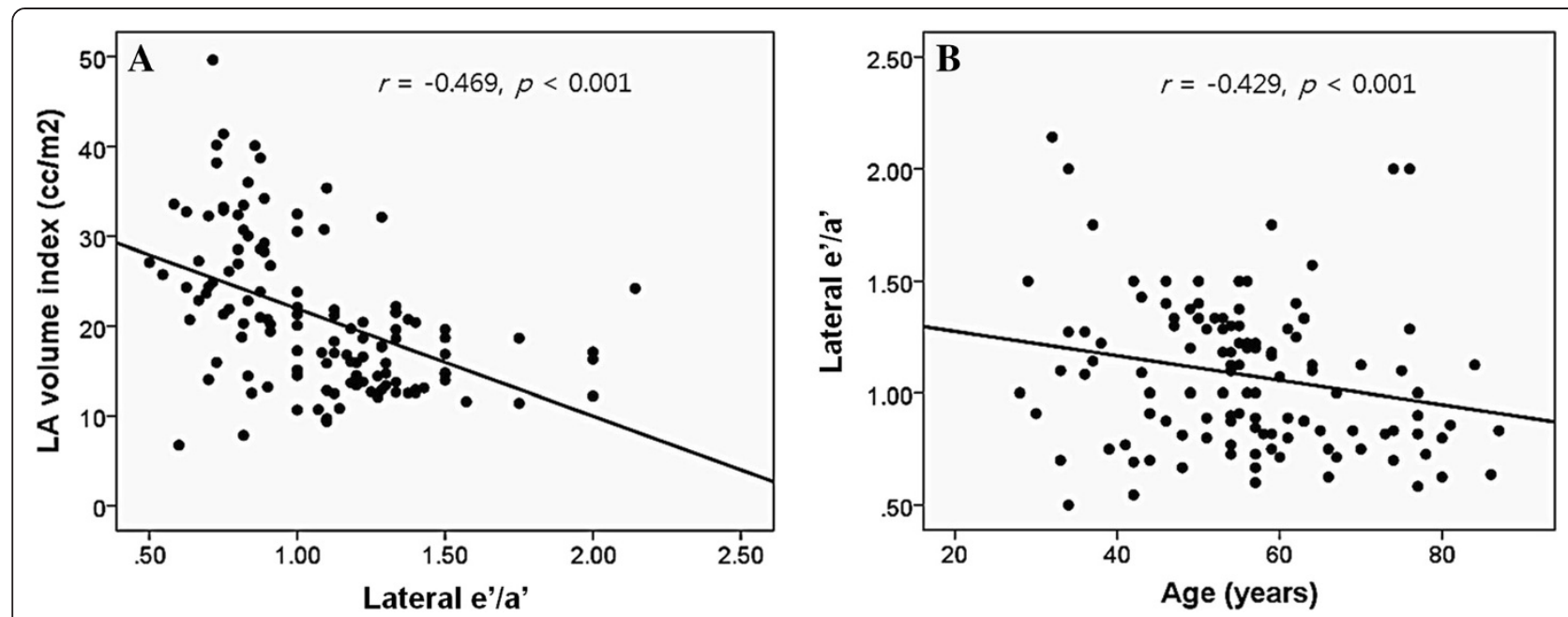

Figure 4 Scatter plots showing the associations of lateral $e^{\prime} / a^{\prime}$ with left atrial volume index (A) and age (B). LA, left atrium. $r$, correlation coefficient.

linear regression analysis showed that lateral e'/a' was independently associated with LAVI $(\beta=-0.484$, $p<0.001)$, even after adjusting for potential confounders including age, sex, body mass index, hypertension and diabetes (Table 2). In the ROC curve analysis, the sensitivity and specificity for detection of LAVI $\geq 34 \mathrm{ml} / \mathrm{m}^{2}$ were $88.9 \%$ and $67.0 \%$, respectively, with lateral $\mathrm{e}^{\prime} / \mathrm{a}^{\prime}$ of 0.89 as the best cut-off value (Figure 5).

Lateral e' has been known to be a maker of diastolic dysfunction [10]. When the data was analyzed using lateral e' velocity instead of lateral e'/a', the lateral e' velocity was significantly lower in subjects with grade II diastolic dysfunction than that in those with normal diastolic function $(7.4 \pm 1.9$ versus $11.6 \pm 2.0 \mathrm{~cm} / \mathrm{s}$, $p<0.001)$. Lateral e' velocity was also negatively associated with LAVI even after adjusting for potential confounders $(\beta=-0.360, p=0.011)$.

The data was analyzed for any correlations between lateral e'/a' and LAVI in subjects with normal diastolic function $(\mathrm{n}=263$ ) and grade I diastolic dysfunction (relaxation abnormality, $n=276$ ). In the univariate analysis, there was a significant correlation between lateral e'/a' and LAVI in subjects with normal diastolic function $(r=-0.348$, $p<0.001$ ), but this was not an independent association

Table 2 Independent associations between left atrial volume index and variables

\begin{tabular}{lccc}
\hline Variable & $\boldsymbol{\beta}$ & $\mathbf{t}$ & $\boldsymbol{p}$ \\
\hline Age & 0.349 & 2.892 & 0.006 \\
Female sex & 0.021 & 0.189 & 0.850 \\
Body mass index & 0.248 & 2.086 & 0.042 \\
Diabetes & 0.136 & 1.245 & 0.219 \\
Hypertension & 0.013 & 0.115 & 0.909 \\
Lateral e'/a' & -0.484 & -4.677 & $<0.001$ \\
\hline
\end{tabular}

as it was not significant in the subsequent multivariable analysis $(\beta=-0.175, p=0.086)$. There were no significant correlations between lateral e'/a' and LAVI in subjects with grad I diastolic dysfunction in both univariate and multivariable analyses.

\section{Discussion}

The present study identified 2 distinct groups of lateral TDI patterns among relatively healthy subjects without structural problems of the heart but with pseudonormal pattern of mitral inflow. Subjects with a concordance pattern between septal and lateral TDI were older, and

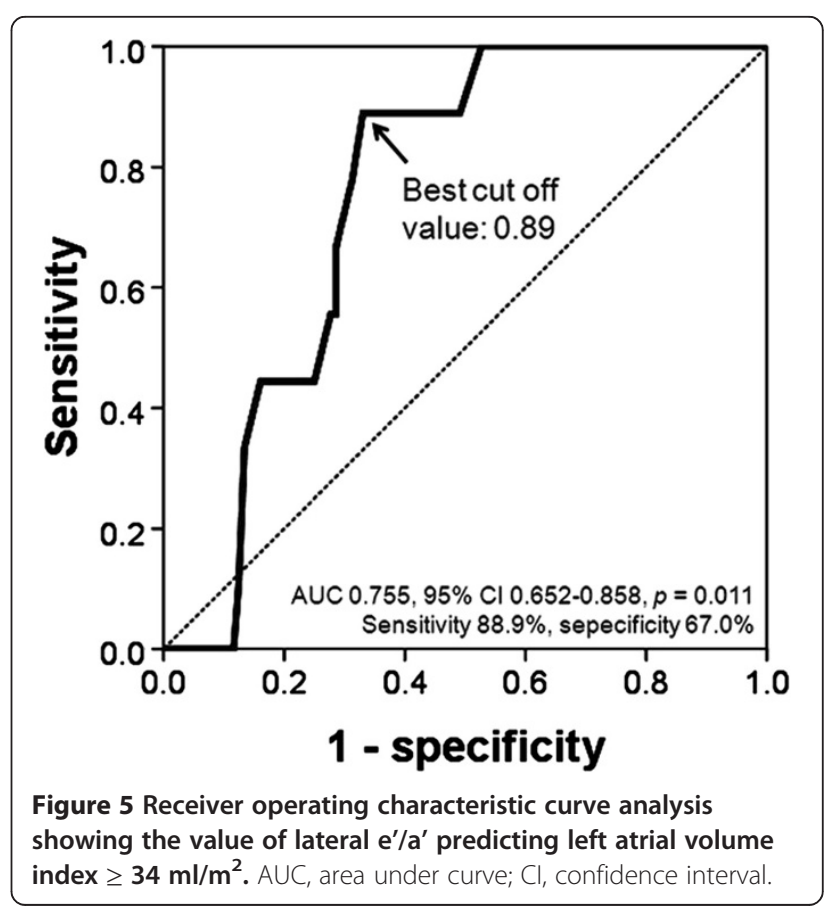


had more unfavorable echocardiographic parameters including high LAVI and E/e', and demonstrated more advanced LV diastolic dysfunction. On the other hand, subjects with a discordance pattern showed nearly normal or mild diastolic dysfunction. Lateral e'/a' had an independent correlation with LAVI even after controlling for potential confounders such as age, sex, body mass index, hypertension and diabetes. This suggests that lateral $e^{\prime} / a^{\prime}$ would be a valuable parameter for assessing diastolic function in subjects with a pseudonormal pattern of mitral inflow.

To date, subjects with pseudonormal pattern of mitral inflow has not been further stratified. These subjects are often considered to have advanced diastolic dysfunction, but the present study have identified that more than half of these subjects $(72 / 122=59 \%)$ have nearly normal diastolic function with small LAVI and low E/e'. We believe that this group of subjects may represent those with "early diastolic dysfunction".

Many different echocardiographic parameters exist in the assessment of diastolic function, and they can help differentiate between normal and pseudonormal patterns of diastolic function [9]. However, each test has limitations such as dependency on loading conditions and image quality [9]. In addition, obtaining appropriate hemodynamic maneuver to differentiate normal and pseudonormal diastolic dysfunctions may be difficult to achieve in all subjects. Moreover, application of several tests is not an easy task in busy clinical settings. In this regard, checking lateral TDI patterns seems to be a simple and useful tool for the diagnosis of LV diastolic function. Measurement of TDI of mitral annulus has been known to be relatively load independent [5] and can be performed in nearly all subjects (95\%), regardless of image quality [9]. In addition, it does not require training of hemodynamic maneuvers to patients or sonographers. From the study results, we can suggest a possible diagnostic pathway for the assessment of LV diastolic function. If a healthy subject without significant cardiac structural abnormality has a pseudonormal pattern of mitral inflow, lateral TDI evaluation can be helpful. With lateral $e^{\prime} / a^{\prime} \geq 1$, a subject can be considered roughly to have no significant diastolic dysfunction but an early diastolic dysfunction.

Our results imply that septal $\mathrm{e}^{\prime} / \mathrm{a}^{\prime}$ is more sensitive marker of diastolic dysfunction compared to lateral e'/a'. Septal e'/a' reversed in the early stage of diastolic dysfunction, however lateral $e^{\prime} / a^{\prime}$ dose not reverse until a more advanced stage of diastolic dysfunction develops. These findings are in line with those of a previous report [8]. Consequently, the severity of diastolic dysfunction is often overestimated especially in subjects with $\mathrm{E} / \mathrm{A} \geq 1$ and septal $\mathrm{e}^{\prime} / \mathrm{a}^{\prime}<1$ unless further tests are performed [14]. Therefore, combination of septal and lateral $e^{\prime} / a^{\prime}$ is useful for the discrimination of early stage of diastolic dysfunction from more advanced stages of diastolic dysfunction among patients with mitral inflow patterns of $\mathrm{E} / \mathrm{A} \geq 1$.

In order to assess the role of lateral e'/a' as a discriminator of diastolic dysfunction, LAVI was used as a reference standard of LV diastolic dysfunction. As commonly observed with TDI, there is an inverse correlation between age and e' velocity and e'/a' $[5,8,13,15]$, and this correlation was also observed in our study (Figure 3). However, we noted that an independent association between lateral $e^{\prime} / a^{\prime}$ and LAVI persisted even when adjusting for confounders including age and other risk factors in a multivariable model.

Because there are various sites for obtaining TDI, a variety of cut-off values for the diagnosis of diastolic dysfunction and/or high LV filling pressure have been suggested. Sohn et al. reported that septal e' $<8.5 \mathrm{~cm} / \mathrm{s}$ and $\mathrm{e}^{\prime} / \mathrm{a}^{\prime}<1$ discriminated a pseudonormal pattern from a normal pattern with a sensitivity of $88 \%$ and a specificity of $67 \%$ [5]. A study by Nagueh et al. demonstrated that lateral E/e' > 10 correlated well with elevated LV filling pressure [16]. Ommen and colleagues reported that $\mathrm{E} / \mathrm{e}^{\prime}<8$ predicted normal LV end-diastolic pressure, and $\mathrm{E} / \mathrm{e}^{\prime}>15$ identified increased LV end-diastolic pressure [7]. Several studies have performed compared the diagnostic utility of TDI at different examination locations. Srivastava et al. compared septal versus lateral TDI and found that septal e' provides better diagnostic performance [17]. In a study of healthy subjects with normal LV systolic function, Firstenberg et al. reported a good correlation between septal e' velocities and LV filling pressures as estimated by invasive hemodynamic study, whereas lateral e' did not show the same correlation [18]. Park and colleagues showed that septal TDI tends to overestimate the severity of LV diastolic dysfunction when compared with lateral TDI, and suggested that lateral TDI measurements more accurately reflects LV diastolic dysfunction than septal TDI measurement do [14]. Other studies have also reported that lateral TDI correlates best with LV filling pressure and with indices of LV stiffness in subjects with normal ejection fraction or coronary artery disease [19-21]. Current guidelines recommend the use of average e' obtained from septal and lateral sides of the mitral annulus for prediction of LV filling pressure [10]. Taken together, to date, there has been no consensus on appropriate selection of annulus for TDI assessment of diastolic function and filling pressure.

\section{Study limitations}

The study sample size is small. Enrolled subjects were healthy without cardiac structural abnormalities, and therefore, the results of the study cannot be generalized 
to other populaitons. The most widely used reference parameter for diastolic function is LV relaxation time; however, LV catheterization was not performed in this study. Other parameters which can be measured during echocardiography, such as mitral inflow pattern with the Valsalva maneuver, pulmonary venous flow pattern and color M-mode propagation velocity, were not evaluated in this study.

\section{Conclusions}

For subjects with $\mathrm{E} / \mathrm{A} \geq 1$ and septal $\mathrm{e}^{\prime} / \mathrm{a}^{\prime}<1$, the severity of diastolic dysfunction is often overestimated. In this case, lateral TDI evaluation is helpful for assessment of diastolic dysfunction. Lateral e' $/ \mathrm{a}^{\prime} \geq 1$ is a valuable indicator of early diastolic dysfunction but not for advanced diastolic dysfunction in this group of people.

\section{Abbreviations}

DT: Deceleration time; HFpEF: Heart failure with preserved ejection fraction; LA: Left atrium; LAVI: Left atrial volume index; LV: Left ventricle; LVEDD: Left ventricular end-diastolic dimension; LVESD: Left ventricular end-systolic dimension; SD: Standard deviation; ROC: Receiver operating characteristics; TDI: Tissue Doppler imaging; TTE: Transthoracic echocardiography.

\section{Competing interests}

The authors declare that they have no competing interests.

\section{Authors' contributions}

The work presented here was carried out in collaboration between all authors. H-LK analyzed data and wrote the paper; J-HZ designed the study, interpreted the results, and had all responsibilities of this work; J-BS, Y-JK, W-YC, S-HK, M-AK and D-WS were involved in study design and interpretation of data. All authors read and approved the final manuscript.

\section{Acknowledgements}

The authors appreciate the help of Seon Kyung Lee, Min-Seon Choi, Yu Nui Gim, Ju Hyun Lee and Seul Ki Lee for data collection.

\section{Author details}

${ }^{1}$ Cardiovascular Center, Seoul National University Boramae Medical Center Seoul, Korea. ${ }^{2}$ Cardiovascular Center, Seoul National University Hospital, Seoul, Korea. ${ }^{3}$ Department of Internal Medicine, Seoul National University Boramae Medical Center, Seoul National University College of Medicine, 39 Boramae-gil, Dongjak-gu, Seoul 156-707, Korea.

Received: 11 July 2013 Accepted: 19 August 2013

Published: 20 August 2013

\section{References}

1. Redfield MM, Jacobsen SJ, Burnett JC Jr, Mahoney DW, Bailey KR, Rodeheffer $\mathrm{RJ}$ : Burden of systolic and diastolic ventricular dysfunction in the community: Appreciating the scope of the heart failure epidemic. JAMA 2003, 289:194-202.

2. Zile MR, Brutsaert DL: New concepts in diastolic dysfunction and diastolic heart failure: Part I: Diagnosis, prognosis, and measurements of diastolic function. Circulation 2002, 105:1387-1393.

3. Galderisi M: Diastolic dysfunction and diastolic heart failure: Diagnostic, prognostic and therapeutic aspects. Cardiovasc Ultrasound 2005, 3:9.

4. Garcia MJ, Thomas JD, Klein AL: New Doppler echocardiographic applications for the study of diastolic function. J Am Coll Cardiol 1998, 32:865-875.

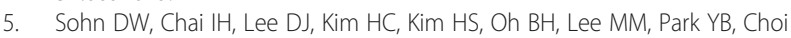
YS, Seo JD, Lee YW: Assessment of mitral annulus velocity by Doppler tissue imaging in the evaluation of left ventricular diastolic function. J Am Coll Cardiol 1997, 30:474-480.

6. Oh JK, Park SJ, Nagueh SF: Established and novel clinical applications of diastolic function assessment by echocardiography. Circ Cardiovasc Imaging 2011, 4:444-455.
7. Ommen SR, Nishimura RA, Appleton CP, Miller FA, Oh JK, Redfield MM, Tajik AJ: Clinical utility of Doppler echocardiography and tissue Doppler imaging in the estimation of left ventricular filling pressures: A comparative simultaneous Doppler-catheterization study. Circulation 2000, 102:1788-1794.

8. Zo JH, Kim SH, Kim MA, Kim YJ, Sohn DW: The change of diastolic tissue Doppler velocity of mitral annulus: the differences between septal and lateral annulus. J Kor Soc Echocardiogr 2004, 12:64-68.

9. Lang RM, Bierig M, Devereux RB, Flachskampf FA, Foster E, Pellikka PA, Picard MH, Roman MJ, Seward J, Shanewise JS, Solomon SD, Spencer KT, Sutton MS, Stewart WJ: Recommendations for chamber quantification: A report from the american society of echocardiography's guidelines and standards committee and the chamber quantification writing group, developed in conjunction with the European association of echocardiography, a branch of the European Society of Cardiology. J Am Soc Echocardiogr 2005, 18:1440-1463.

10. Nagueh SF, Appleton CP, Gillebert TC, Marino PN, Oh JK, Smiseth OA, Waggoner AD, Flachskampf FA, Pellikka PA, Evangelisa A:

Recommendations for the evaluation of left ventricular diastolic function by echocardiography. Euro J Echocardiogr 2009, 10:165-193.

11. Schiller NB, Shah P, Crawford M, DeMaria A, Devereux R, Feigenbaum H, Gutgesell H, Reichek N, Sahn D, Schnittger I: Recommendations for quantitation of the left ventricle by two-dimensional echocardiography. American Society of Echocardiography committee on standards, subcommittee on quantitation of two-dimensional echocardiograms. J Am Soc Echocardiogr 1989, 2:358.

12. Moller JE, Hillis GS, Oh JK, Seward JB, Reeder GS, Wright RS, Park SW, Bailey KR, Pellikka PA: Left atrial volume: A powerful predictor of survival after acute myocardial infarction. Circulation 2003, 107:2207-2212.

13. Tighe DA, Vinch CS, Hill JC, Meyer TE, Goldberg RJ, Aurigemma GP: Influence of age on assessment of diastolic function by Doppler tissue imaging. Am J Cardiol 2003, 91:254-257.

14. Park HS, Naik SD, Aronow WS, Visintainer PF, Das M, McClung JA, Belkin RN: Differences of lateral and septal mitral annulus velocity by tissue Doppler imaging in the evaluation of left ventricular diastolic function. Am J Cardiol 2006, 98:970-972.

15. Onose Y, Oki T, Mishiro Y, Yamada H, Abe M, Manabe K, Kageji Y, Tabata T, Wakatsuki T, Ito S: Influence of aging on systolic left ventricular wall motion velocities along the long and short axes in clinically normal patients determined by pulsed tissue Doppler imaging. J Am Soc Echocardiogr 1999, 12:921-926.

16. Nagueh SF, Middleton K, Kopelen HA, Zoghbi WA, Quinones MA: Doppler tissue imaging: A noninvasive technique for evaluation of left ventricular relaxation and estimation of filling pressures. J Am Coll Cardiol 1997, 30:1527-1533.

17. Srivastava PM, Burrell LM, Calafiore P: Lateral vs medial mitral annular tissue Doppler in the echocardiographic assessment of diastolic function and filling pressures: Which should we use? Euro J Echocardiogr 2005, 6:97-106.

18. Firstenberg MS, Levine BD, Garcia MJ, Greenberg NL, Cardon L, Morehead AJ, Zuckerman J, Thomas JD: Relationship of echocardiographic indices to pulmonary capillary wedge pressures in healthy volunteers. J Am Coll Cardiol 2000, 36:1664-1669.

19. Kasner M, Westermann D, Steendijk P, Gaub R, Wilkenshoff U, Weitmann K, Hoffmann W, Poller W, Schultheiss HP, Pauschinger M, Tschope C: Utility of Doppler echocardiography and tissue Doppler imaging in the estimation of diastolic function in heart failure with normal ejection fraction: A comparative Doppler-conductance catheterization study. Circulation 2007, 116:637-647.

20. Rivas-Gotz C, Manolios M, Thohan V, Nagueh SF: Impact of left ventricular ejection fraction on estimation of left ventricular filling pressures using tissue Doppler and flow propagation velocity. Am J Cardiol 2003, 91:780-784.

21. Galderisi M, Rapacciuolo A, Esposito R, Versiero M, Schiano-Lomoriello V, Santoro C, Piscione F, de Simone G: Site-dependency of the E/e' ratio in predicting invasive left ventricular filling pressure in patients with suspected or ascertained coronary artery disease. Eur Heart $J$ Cardiovascular Imaging 2013, 14:555-561.

doi:10.1186/1476-7120-11-31

Cite this article as: Kim et al:: Additional value of lateral tissue Doppler imaging in the assessment of diastolic dysfunction among subjects with pseudonormal pattern of mitral inflow. Cardiovascular Ultrasound 2013 11:31. 